\title{
Can we prevent significant brain desaturation during defibrillator testing by increasing the brain saturation reserve?
}

\author{
Tina Hu, MD, MSc • Anne Lavoie, MD • Alain Deschamps, MD, PhD • Peter Guerra, MD • \\ Denis Babin, MSc · Annik Fortier, MSc $\cdot$ Jean Lambert, PhD $\cdot$ André Y. Denault, MD, PhD
}

Received: 13 December 2017/ Accepted: 25 December 2017/Published online: 19 January 2018

(C) Canadian Anesthesiologists' Society 2018

\section{To the Editor,}

In patients undergoing electrophysiology procedures, programmed threshold testing for internal cardioverter and defibrillator (ICD) insertion involves testing of the apparatus and repeated inductions of ventricular fibrillation (VF), which can result in global cerebral hypoperfusion similar to cardiac arrest. ${ }^{1}$ Hypercapnia is known to increase cerebral blood flow (CBF) and cerebral vasodilation. The adjustment of ventilation to raise cerebral regional saturation $\left(\mathrm{rSO}_{2}\right)$ is a common intervention in

Tina Hu and Anne Lavoie: co-first authorship.

T. Hu, MD, MSc · A. Deschamps, MD, PhD - D. Babin, MSc . A. Y. Denault, MD, PhD ( $\square)$

Department of Anesthesiology, Montreal Heart Institute,

Université de Montréal, Montreal, QC, Canada

e-mail: andre.denault@gmail.com

T. $\mathrm{Hu}, \mathrm{MD}, \mathrm{MSc}$

Faculty of Medicine, University of Toronto, Toronto, ON, Canada

A. Lavoie, MD

Department of Anesthesiology, Centre Hospitalier de

l'Université de Montréal, Montreal, QC, Canada

P. Guerra, MD

Department of Cardiology, Montreal Heart Institute, Université de Montréal, Montreal, QC, Canada

A. Fortier, MSc

Montreal Health Innovations Coordinating Center, Montreal

Heart Institute, Montreal, QC, Canada

J. Lambert, $\mathrm{PhD}$

Department of Social and Preventive Medicine, School of Public

Health, Université de Montréal, Montreal, QC, Canada correcting brain desaturation and part of a previously reported and validated algorithm. ${ }^{2}$

Preliminary evidence has shown that hypoventilation (with resulting hypercarbia) may prevent cerebral desaturations during ICD insertion and cardiac arrest. ${ }^{3}$ It is possible that deeper sedation with increased hypoventilation and hypercapnia may increase CBF to compensate for decreases in cardiac output associated with $\mathrm{VF}$ and reduce the risk of cerebral desaturation. The use of near-infrared spectroscopy (NIRS) to monitor and prevent brain desaturation by pre-emptively increasing CBF has not been reported. The purpose of this study is to test the effectiveness of permissive sedation-induced hypercapnia in decreasing the severity of cerebral desaturation episodes during ICD insertion.

This randomized-controlled study was approved (032009 \#03-076) by the Montreal Heart Institute ethics review board. After informed consent, all patients undergoing ICD insertion were sedated with remifentanil and propofol and randomized in two groups: a "light sedation" (LS) and a "deep sedation" (DS) group. Monitoring using NIRS and blood gases was performed in both groups. The VF test was induced via preprogrammed electrical stimulations. The primary endpoint was a reduction in episodes of significant cerebral desaturation in the group with permissive hypercapnia. Unfortunately, the trial was stopped before completion because, unexpectedly, recommendations were made to avoiding testing the defibrillators. ${ }^{4} \mathrm{~A}$ total of 13 (out of 20) patients were recruited for the study with six LS and six DS patients and one exclusion. On average, patients were older in the LS than DS group (69 yr vs $58 \mathrm{yr}$ ) and more frequently males (LS: six males; DS: two males/four females). The DS group had lower mean (standard deviation [SD]) $\mathrm{pH}$ [7.35 (0.01), DS vs 7.43 (0.04), L; $P=$ 


\section{Control ("Light Sedation") Group Intervention ("Deep Sedation") Group}

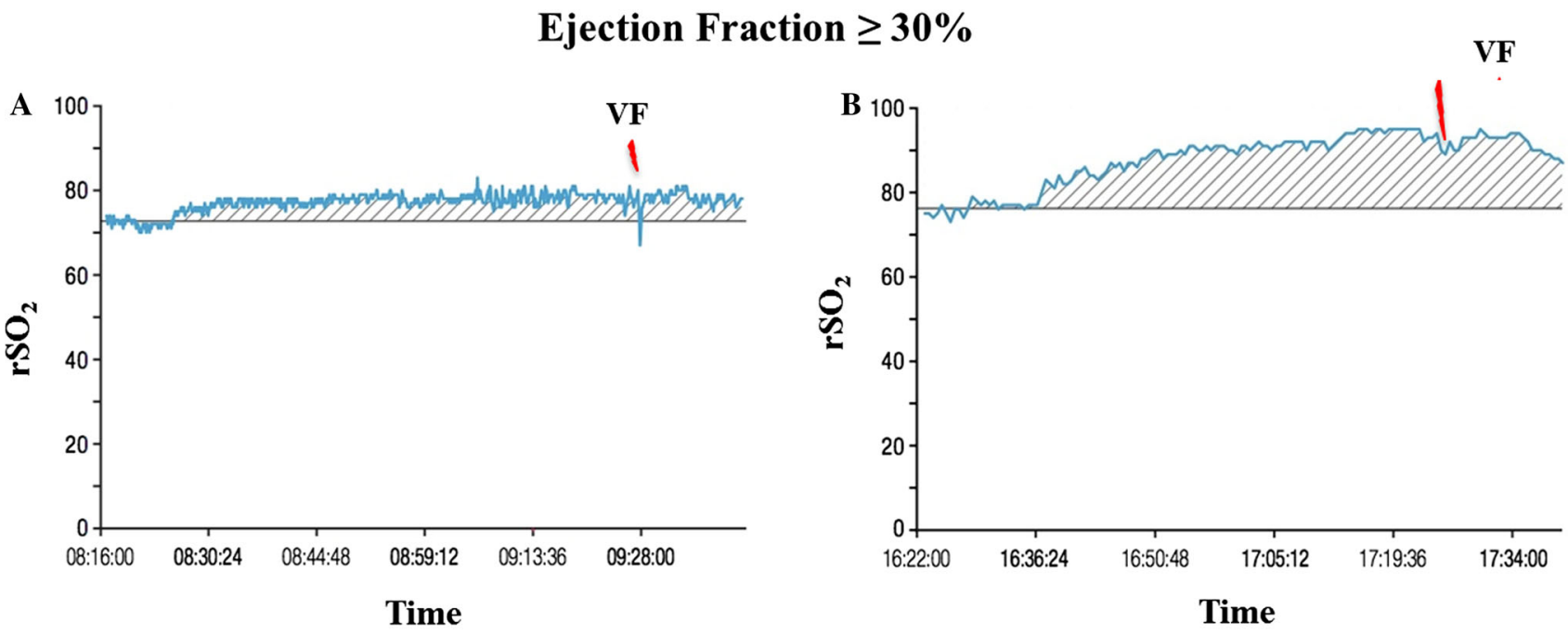

\section{Ejection Fraction $<30 \%$}

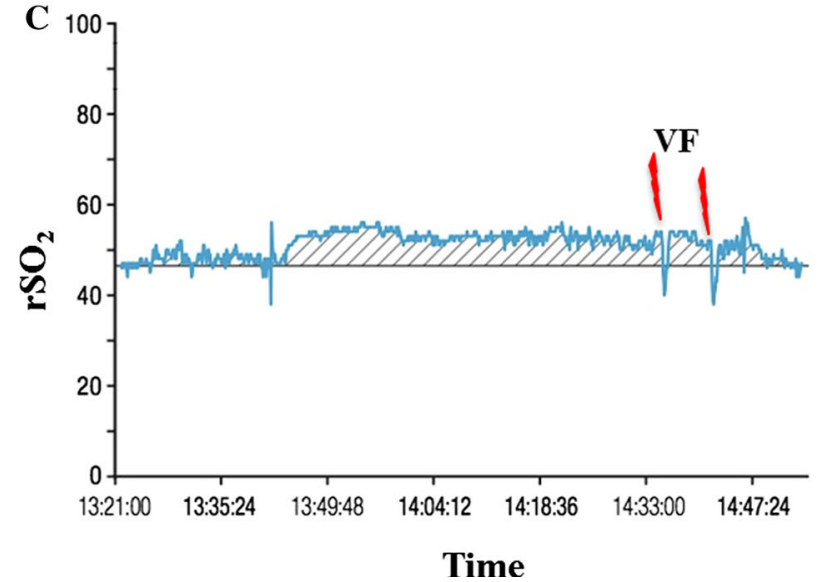

Figure Example of four patients, two with "light sedation" (LS) (A, C) and two with "deep sedation" (DS) (B, D), undergoing internal cardioverter and defibrillator (ICD) insertion and repeated inductions of ventricular fibrillation (VF). Patients A and B had ejection fractions $\geq 30 \%$ with a baseline cerebral regional saturation $\left(\mathrm{rSO}_{2}\right)$ above $70 \%$. Note that patient in the DS group (B) had a proportionally higher area over the baseline cerebral regional saturation $\left(\mathrm{rSO}_{2}\right)$ value compared with an LS patient (A) before VF, which was associated with a

0.006] and higher mean (SD) partial pressure of carbon dioxide $\left(\mathrm{PaCO}_{2}\right)$ [50 (5), DS vs 34 (3) $\mathrm{mmHg}$, LS; $P<$ $0.001]$ prior to $\mathrm{VF}$ and higher remifentanil infusion rates compared with controls $(P=0.03)$. There were no significant differences between groups in terms of propofol infusion rates, total duration of $\mathrm{VF}$, number of VFs, and number of shocks. Ventricular fibrillation was associated with a reduction in cerebral $\mathrm{rSO}_{2}$ values in all patients. Brief desaturations after VF below baseline occurred in three DS patients $(50 \%)$ and in five LS patients $(83 \%)$. The area over the baseline cerebral $\mathrm{rSO}_{2}$

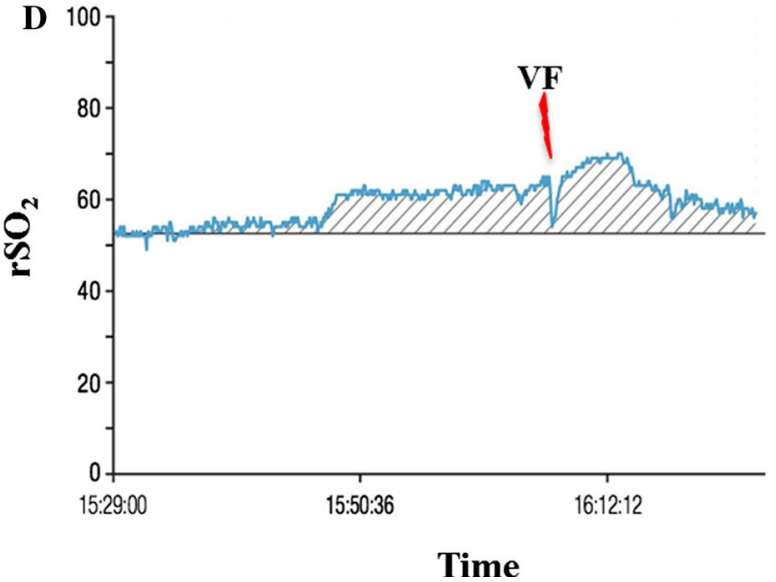

reduction in the cerebral $\mathrm{rSO}_{2}$ value, but it only fell below the $\mathrm{rSO}_{2}$ baseline in the LS group. Patients $\mathrm{C}$ and $\mathrm{D}$ had reduced ejection fractions $<30 \%$ with a baseline $\mathrm{rSO}_{2}$ below $60 \%$. Note that the DS patient (D) had a significantly higher area over the baseline cerebral $\mathrm{rSO}_{2}$ value compared with the LS patient (C). Ventricular fibrillation was associated with a reduction in cerebral $\mathrm{rSO}_{2}$ values in both patients, but it only fell below the $\mathrm{rSO}_{2}$ baseline in the LS group

values was higher in the DS compared with LS group [left: 1241 (463), DS vs 303 (124) \%.min, LS; $P=0.004$; and right: 1282 (490), DS vs 259 (108) \%.min, LS; $P=0.003$ ], even when corrected for procedural time $(P<0.001)$. The Figure illustrates four patients. Patients with lower ejection fractions (EF) had reduced baseline $\mathrm{rSO}_{2}$ values compared with those with normal EF. Ventricular fibrillation was associated with brief episodes of desaturation in both groups but it remained above baseline in the DS group because of the increased saturation reserve. 
These observations are pertinent for the increased role of NIRS in cardiac arrest ${ }^{5}$ suggesting that patients with good resuscitation outcomes have significantly higher NIRS values. Deeper sedation and consequently a higher $\mathrm{PaCO}_{2}$ value increase $\mathrm{rSO}_{2}$ and may attenuate the reduction in $\mathrm{rSO}_{2}$ and consequently cerebral blood flow during VF.

Disclosures Dr. Denault is on the Speakers Bureau for CAEHealthcare, Edwards, Masimo, and Medtronic.

Conflicts of interest None declared.

Editorial responsibility This submission was handled by Dr. Philip M. Jones, Associate Editor, Canadian Journal of Anesthesia.

Funding sources Supported by the Montreal Heart Institute Foundation.

\section{References}

1. Karaoguz R, Altln T, Atbasoglu EC, et al. Defibrillation testing and early neurologic outcome. Int Heart J 2008; 49: 553-63.

2. Deschamps A, Hall R, Grocott $H$, et al. Cerebral oximetry monitoring to maintain normal cerebral oxygen saturation during high-risk cardiac surgery: a randomized controlled feasibility trial. Anesthesiology 2016; 124: 826-36.

3. Meex I, Dens J, Jans F, et al. Cerebral tissue oxygen saturation during therapeutic hypothermia in post-cardiac arrest patients. Resuscitation 2013; 84: 788-93.

4. Healey JS, Hohnloser SH, Glikson M, et al. Cardioverter defibrillator implantation without induction of ventricular fibrillation: a single-blind, non-inferiority, randomised controlled trial (SIMPLE). Lancet 2015; 385: 785-91.

5. Parnia S, Yang J, Nguyen $R$, et al. Cerebral oximetry during cardiac arrest: a multicenter study of neurologic outcomes and survival. Crit Care Med 2016; 44: 1663-74. 